\title{
Midazolam for sedation in the paediatric intensive care unit
}

\author{
D. A. Rosen and K. R. Rosen
}

Section of Pediatric Anesthesiology, Department of Anesthesiology, C4139 Med Inn Bridge, Box 0800, University of Michigan Medical Center, 1500 E. Medical Center Drive, Ann Arbor, Michigan 48109-0800, USA

\begin{abstract}
This retrospective study examines data from 55 patients sedated in a paediatric intensive care unit (PICU) with midazolam. Midazolam sedation was initiated with a bolus of $0.25 \mathrm{mg} \cdot \mathrm{kg}^{-1}$ followed by a continuous infusion of $0.4-4 \mu \mathrm{g} \cdot \mathrm{kg}^{-1} \cdot \mathrm{min}^{-1}$. Physiological and metabolic parameters, infusion rates, duration, and sedation scores were monitored. Midazolam infusions were effective in sedating all the children studied during all or part of their PICU admission. The median duration of sedation was $74 \mathrm{~h}$ with a range of 4 to $1272 \mathrm{~h}$. Haemodynamics were unchanged. Of the patients $46 \%$ were effectively alimented by the enteral route, and enteral alimentation was successful in all patients in whom it was attempted. Unassisted ventilation occurred in $44 \%$ of the patients during infusion. Oxygen consumption was $28 \%$ lower than in the control. Disadvantages of midazolam infusion have included inability to sedate during extracorporeal membrane oxygenation and development of acute tolerance.
\end{abstract}

Key words: Hypnotics - Benzodiazepines - Intensive care - Paediatrics - Sedation - Continuous infusion

The paediatric intensive care unit (PICU) is a stress-filled environment. In the course of confronting life-threatening illness, a child in the PICU encounters many sources of physical and emotional distress, including invasive and painful procedures, separation from family and home, and fear of strange people, sounds, and equipment. The child's ability to cooperate with and accept medical therapy may profoundly influence outcome. Sedative medications are important adjuvants in the care of critically ill children. However, there is little information about the safe and reliable sedation of children in the PICU or in any other setting [1].

The ideal sedative does not exist. Midazolam, however, possesses more of the properties of this ideal than do the traditional sedatives (narcotics, barbiturates, or phenothiazines). The benzodiazepines are the only class of drugs that specifically reduce patient anxiety. Midazolam is distinguished among the benzodiazepines by its water solubility, rapid onset, short duration, and lack of accumulation or active metabolites [2]. These pharmacokinetic characteristics support its use by continuous infusion. This paper presents a retrospective study that describes our experience with continuous intravenous infusion of midazolam in the PICU.

\section{Methods}

\section{Sedation protocol}

Any child presenting to the PICU who required continuous sedation was considered for midazolam infusion. Midazolam infusions were initiated under a protocol we have described previously [3]. Sedation was initiated with a slow intravenous bolus of $0.25 \mathrm{mg} \cdot \mathrm{kg}^{-1}$ over $3-5 \mathrm{~min}$. The continuous intravenous infusion was started immediately at a rate of $0.4 \mu \mathrm{g} \cdot \mathrm{kg}^{-1} \cdot \mathrm{min}^{-1}$. The initial range for the infusion rate was $0.4-2 \mu \mathrm{g} \cdot \mathrm{kg}^{-1} \cdot \mathrm{min}^{-1}$ but was increased to $4.0 \mu \mathrm{g} \cdot \mathrm{kg}^{-1} \cdot \mathrm{min}^{-1}$ after $48 \mathrm{~h}$ if necessary. The dose was titrated to maintain a sedation score of 3. The infusion was decreased when a score of 2 was reached and increased at a score of 4 . Analgesics were prescribed for the treatment of pain when indicated. The use of muscle relaxants or of any medications with sedative properties was noted.

\section{Patient monitoring}

Demographic information collected for each patient included age, weight, medical, surgical, allergic, and medication histories, and PICU admission diagnosis. The midazolam bolus dose and mean hourly infusion rates were recorded. The total duration of infusion was noted. Vital signs (blood pressure, heart rate, temperature, respiratory rate, and oxygen saturation when indicated) were also recorded every hour. The use of ventilatory support was described in detail. Alimentation, either enteral or parenteral, was also noted. In a limited number of patients, metabolic parameters, respiratory quotient, oxygen consumption, and carbon dioxide production were measured with the Cybermedic Metabolic Monitor, both before and after sedation of the patient.

\section{Evaluation of sedation}

The effectiveness of the sedation was rated on a 5 -point activity scale each hour (Table 1). A comatose patient received a score of 1; the agitated and struggling patient was given a score of 5 . A score greater than 3 
Table 1. Five-point activity scale for sedation

\begin{tabular}{ll}
\hline Score & Level of activity \\
\hline 1 & Comatose; does not respond to stimulation \\
2 & Asleep; awakens with stimulation \\
3 & Calm; catheters not at risk \\
4 & Fussy; catheters at risk \\
5 & Wild; no control; thrashing \\
\hline
\end{tabular}

defined inadequate sedation, requiring modification of sedative treatment either by increase in midazolam infusion rate or by use of alternative sedative medication.

\section{Results}

Midazolam infusions were successfully employed for sedation in 55 children of all ages. The youngest patient in this series was a child born at 32 weeks, who was 5 weeks of age at the start of infusion. The median age was 2.5 years (Table 2). The median weight was $11.5 \mathrm{~kg}$, with a range of $2-75 \mathrm{~kg}$. The admission diagnosis for the majority of patients was respiratory insufficiency (Table 3 ). The primary indication for use of a midazolam infusion was maintenance of an endotracheal tube with or without spontaneous ventilation.

This sedation protocol was successful for all children on whom it was attempted. The median duration of midazolam infusion was $74 \mathrm{~h}$, with a range of $4-1272 \mathrm{~h}$. The mean infusion dose was $0.92 \pm 0.54 \mu \mathrm{g} \cdot \mathrm{kg}^{-1} \cdot \mathrm{min}^{-1}$ (Fig. 1). There was no correlation between age and mean infusion rate $\left(r^{2}=2.19 \times 10^{-4}\right)$. In those patients who demonstrated agitation during midazolam infusion (with sedation scores of $4-5$ ), the period of inadequate sedation was less than $10 \%$ of the total infusion time. The majority of patients experienced adequate sedation throughout the time of midazolam infusion. The most commonly utilized supplemental medications were chloral hydrate and morphine. Fig. 2 shows the proportion of patients requiring supplemental medications and the number of medications they received.

One patient was noted to have hallucinations and tremours that occurred $48 \mathrm{~h}$ after abrupt discontinuation of an infusion that had been at $2 \mu \mathrm{g} \cdot \mathrm{kg}^{-1} \cdot \mathrm{min}^{-1}$ for a month. In the current series $45 \%$ of children received some sedative medication following discontinuation of the midazolam infusion. This usually occurred over a period of $4-48 \mathrm{~h}$ post infusion.

No adverse respiratory effects were observed with the use of midazolam. Of the children $44 \%$ had periods of spontaneous respiration during their midazolam infusion. In these spontaneously breathing paediatric patients, normocarbia, as reported by serial arterial blood gases, was maintained. The midazolam infusion was discontinued in most patients at the time of extubation.

Three patients underwent metabolic studies. There was a mean $28 \%$ reduction in oxygen consumption, a $5 \%$ decrease in $\mathrm{CO}_{2}$ production, and a $5 \%$ rise in the respiratory quotient following initiation of the midazolam infusion. Cardiovascular instability was not shown to develop or worsen as a result of the use of

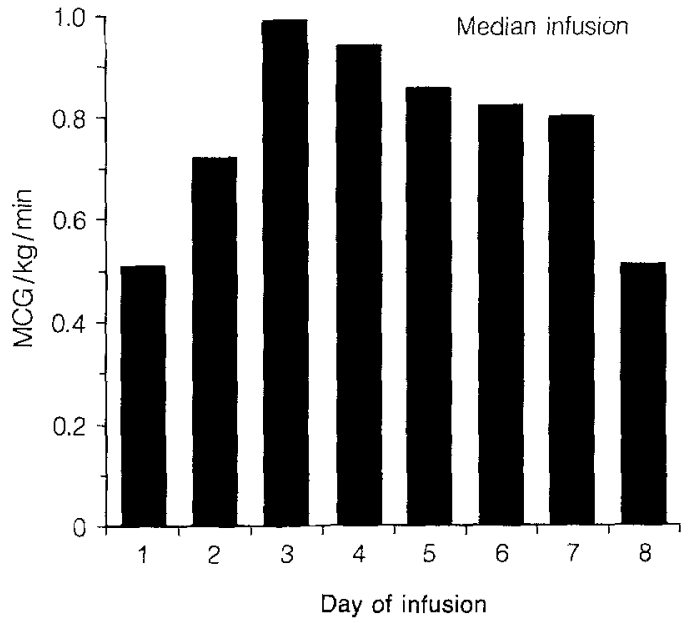

Fig. 1. Median infusion rate over time

Table 2. Age composition of sample

\begin{tabular}{lr}
\hline Age (years) & $n$ \\
\hline $0-1$ & 17 \\
$1-2$ & 15 \\
$2-3$ & 7 \\
$3-4$ & 3 \\
$4-5$ & 3 \\
$8-11$ & 2 \\
$11-14$ & 2 \\
$15-19$ & 6 \\
\hline
\end{tabular}

Table 3. (Admission) diagnosis of patients sedated with midazolam infusion

\begin{tabular}{lc}
\hline Diagnosis & Number of patients \\
\hline Respiratory & 37 \\
Sepsis & 8 \\
Circulatory & 7 \\
Neurologic & 2 \\
Trauma & 2 \\
Haematologic & 1 \\
\hline
\end{tabular}

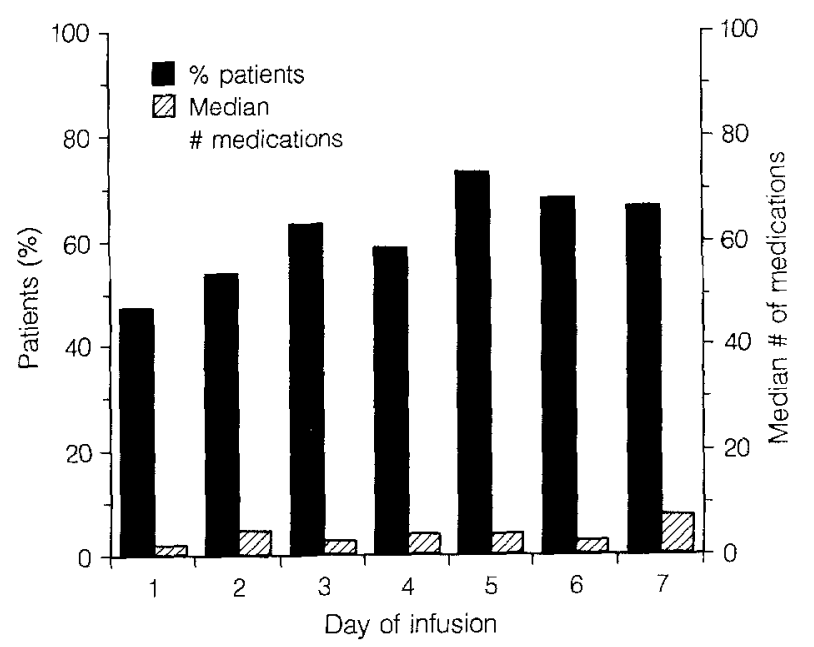

Fig. 2. Percent of patients requiring supplemental medications and median number of doses 


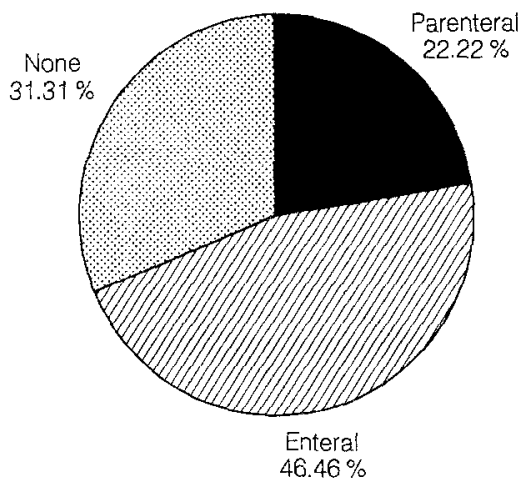

Fig. 3. Route of alimentation utilised in children during midazolam infusion

midazolam infusion. Blood pressure and heart rate remained within $10 \%$ of baseline values. For those patients who were receiving inotropic therapy no increases in these drugs were needed when the midazolam infusion was initiated. Although a history of seizures was common in our patients, no seizures were observed during the infusion. All children in whom enteral alimentation was attempted could be fed through the GI tract (Fig. 3) and $46 \%$ of the children received enteral nutrition during the midazolam infusion.

The one limitation of midazolam discovered during the sedation protocol was the introduction of extracorporeal membrane oxygenation (ECMO). A 1-year-old patient who had been well sedated with the midazolam infusion before initiation of ECMO became acutely agitated when ECMO was begun. Subsequently, he was unable to be sedated with midazolam during ECMO despite an increase in dosage.

\section{Discussion}

Cooperation with medical care is the major reason for sedation in the PICU. In the past this cooperation had been accomplished through the use of intermittent morphine, chloral hydrate, and long-acting benzodiazepine. The ineffectiveness of this approach was confirmed by the use of muscle relaxants in over $90 \%$ of our ICU patients. This midazolam sedation protocol was introduced into our PICU 3 years ago and has become the most popular form of continuous sedation. It is thought to provide more reliable sedation than other techniques and the use of muscle relaxants has now fallen to $65 \%$. Unfortunately, it is difficult to study sedation, because there are no validated tools for measuring sedation objectively in paediatric patients. We did not compare the study protocol with any other regimen because we did not have a satisfactory alternative. Most of the sedative protocols used previously in our PICU involved muscle relaxants or intermittent boluses of medications, used when the patients became uncontrollable.

We found that the midazolam infusion produced the desired effect in the great majority of situations in which it had been attempted. We did not measure midazolam levels because these have been found to correlate poorly with sedation [4]. Success was quantified through the use of sedation scores and the need for additional medications to achieve cooperation. Older children described amnesia for the duration of the midazolam infusion. Our infusion protocol employs a different dosage regiment than is commonly used for adults [5]. The infusion rate is kept low during the first $48 \mathrm{~h}$ because we feel that breakthrough is typically due to something that should be controlled by other medications. Typically this is pain, and an analgesic should be employed rather than an increase in the infusion rate.

\section{Advantages}

Midazolam produced minimal physiological alteration when administered according to our protocol in paediatric patients. Though reports of myocardial and respiratory depression are found in the adult literature [6], cardiovascular variables did not change in these patients, even in those who required inotropic support before and during the midazolam infusion. Likewise, midazolam infusion did not induce respiratory depression in any child. Normal spontaneous ventilation with normocarbia could be maintained in the absence of pulmonary disease. Midazolam infusion was usually discontinued at the time of tracheal extubation. Indicators of the metabolic state were measured in a limited number of patients. A decrease in total body oxygen consumption as well as $\mathrm{CO}_{2}$ production and an increase in the respiratory quotient were noted during midazolam infusion. The calorific requirements of a critically ill child can be quite elevated and the ability to maintain adequate nutrition is essential. Traditional methods of sedation that employ opiates or muscle relaxants frequently require parenteral forms of alimentation. Because a central venous catheter is often not required in a PICU admission these children will be denied the benefits of adequate nutrition during all or part of their ICU admission. Midazolam infusions, by allowing enteral alimentation, are superior in this respect.

Continuous intravenous infusions are preferable to intermittent bolus therapies because of the moment-to-moment control and flexibility attained at a lower total dose of drug. They also eliminate the peaks and troughs associated with intermittent use. Therefore, the occurrence of side effects or frequent periods of inadequate sedation is minimized.

When inadequate sedation is observed it is important to rule out other causes of agitation, such as hypoxia and inadequate ventilation. For this reason continuous monitoring of oxygen saturation is essential in children undergoing this continuous sedation technique.

There is also great flexibility in the route of midazolam administration. Besides intravenous infusion, $a b-$ sorption from mucosal surfaces - oral, nasal, gastric, or rectal - is also dependable. A dose of midazolam may be given by any of these methods if intravenous access is interrupted. Intranasal administration is often chosen because gastrointestinal function is not an issue and uptake is rapid, allowing onset of sedation to occur in 3-10 min [7]. We have also given the drug orally when intravenous access was not available. The onset from oral sedation re- 
quires 30-45 $\mathrm{min}$. We have no personal experience with rectal administration of midazolam, though it does not appear to be superior to the intranasal or oral route.

Some of the beneficial qualities of midazolam are even more difficult to quantify. Preservation of a relatively normal sleep-wakefulness cycle was noted in our patients, although disorders of sleep pattern are not uncommon in an intensive care unit. Comments from nurses and families were overwhelmingly positive. Parents enjoyed being able to maintain some form of communication with their child, even if it was only a reassuring touch. The administration of medication by continuous infusion is also an optimal use of nursing skill. On a daily basis it allows the time spent obtaining and delivering these controlled substances to be spent in direct patient care. The nurses in our unit appreciated the ease of titration and the ability to adjust for different levels of stimulation throughout the day.

\section{Limitations}

Although the use of midazolam is a substantial improvement in the field of sedation, it falls short of the ideal. The major disadvantage intrinsic to all benzodiazepines is the great variability in the clinical pharmacodynamic effect despite dependable pharmacokinetics. Midazolam has a significantly shorter half-life than other benzodiazepines but still requires $1-4 \mathrm{~h}$ for dissipation of effect following discontinuation of therapy.

The preservative in Versed (midazolam) is benzyl alcohol. Attention has recently been focused on the exposure of very small, immature infants to benzyl alcohol and its role in producing kernicterus [8]. Caution must be observed when infants are exposed to several concurrent sources of benzyl alcohol.

Midazolam is distinguished from the other sedatives and even from other benzodiazepines by its low potential for the development of tolerance or addiction. Maintenance infusion requirements and the need for supplemental medications frequently showed a steady decrease during long-term continuous infusion therapy (Fig. 3). Acute tolerance was evident in 2 children, who received infusion doses 10 times greater than anticipated. The infusion was started at $4.0 \mu \mathrm{g} \cdot \mathrm{kg}^{-1} \cdot \mathrm{min}^{-1}$. These children were initially well sedated without respiratory or cardiovascular compromise, but became increasingly difficult to sedate after the second day of continuous infusion. When the medication error was detected, these children were receiving over $6 \mu \mathrm{g} \cdot \mathrm{kg}^{-1} \cdot \mathrm{min}^{-1}$. A decision was made to discontinue the infusion. No evidence of a withdrawal reaction was apparent even after the modest overdose.

Dependence and withdrawal responses are difficult to assess in a child, especially in the PICU. Disorientation and environmental psychosis is noted in many long-term occupants, although altered mental status may be inherent in the primary disease process. Children may lack the verbal skills necessary to differentiate the symptoms of withdrawal from the multiple sources of stress present in the PICU. Our experience of three years has produced only one patient in whom signs and symptoms of an acute withdrawal reaction were suspected. This child was a 15-year-old male oncology patient with widespread metastatic disease who had been on a midazolam infusion for $790 \mathrm{~h}$. He was inadvertently extubated and the midazolam and fentanyl infusion were abruptly discontinued in an effort to avoid reintubation. He developed tremours and hallucinations soon after this acute withdrawal of medication. His symptoms intensified over the next $48 \mathrm{~h}$ and were not relieved by phenothiazine (haloperidol) therapy. Morphine and diazepam did not control his symptoms and he was restarted on a midazolam infusion. There were many potential explanations of these symptoms, including hepatic encephalopathy, respiratory insufficiency, prolonged PICU residence, narcotic withdrawal, or even benzodiazepine withdrawal. This isolated incident with a probable multifactorial aetiology has not altered our protocol. We do not employ gradual weaning regimens.

One area of concern is the apparent interaction of midazolam with the oxygenator commonly used for ECMO. In isolated membrane studies, the SciMed brand oxygenator was capable of absorbing more than $11 \mu \mathrm{g} \cdot \mathrm{cm}^{-2}$ of membrane surface area $[9,10]$. This capacity may explain the difficulties encountered in sedating children on ECMO.

\section{Interactions}

Midazolam has no analgesic qualities and analgesic therapy will be required for control of pain. Analgesic therapy, like hypoxia and hypercarbia, should be considered whenever there is a breakthrough in sedation. Caution must be exercised when narcotics are administered together with midazolam. Synergism is reported with this combination [11]. Midazolam can intensify not only the analgesic properties but also the sedation and the potential for respiratory depression.

\section{Summary}

Now that we have identified an effective method for continuous sedation, well-controlled prospective studies will need to be performed to compare this technique with others. In light of the frequent discordances between pharmacokinetic variables and pharmacodynamic responses, a vital part of this process will be the development of a validated scoring system for measuring paediatric sedation accurately.

Clinical satisfaction and acceptance have resulted in a steady expansion of applications. When our protocol has been followed, we have observed minimal adverse side effects, maintenance of enteral function, and lack of respiratory depression. A reliable level of sedation and cooperation that can be readily adjusted to meet the dynamically fluctuating demands of the PICU was noted. This regimen has become the predominant method of sedation or control in our PICU.

\section{References}

1. Committee on Drugs, Section on Anesthesiology, American Academy of Pediatrics (1985) Guidelines for the elective use of conscious 
sedation, deep sedation and general anesthesia in pediatric patients. Pediatrics 76:317-321

2. Reves JG, Fragen RJ, Vinik R, Greenblatt D (1985) Midazolam: pharmacology and uses. Anesthesiology 62:310-324

3. Silvasi DL, Rosen DA, Rosen KR (1988) Continuous infusion of midazolam for sedation in the PICU. Anesth Analg 67:286-288

4. Booker PD, Beechey A, Lloyd-Thomas AR (1986) Sedation of children requiring artificial ventilation using an infusion of midazolam. Br J Anaesth 58:1104-1108

5. Shapiro JM, Westphal LM, White PF, Sladen RN, Rosenthal MH (1986) Midazolam infusion for sedation in the intensive care unit: effect on adrenal function. Anesthesiology 64:394-398

6. Reves JG, Kissin I, Fournier S (1984) Negative inotropic effects of midazolam (letter). Anesthesiology 60:517-518

7. Wilton NCT, Leigh J, Rosen D, Pandit U (1988) Preanesthetic sedation of preschool children using intranasal midazolam. Anesthesiology 69:1972-1975

8. Jardine DS, Rogers K (1989) Relationships of benzyl alcohol to kernicterus, intraventricular hemorrhage, and mortality in preterm infants. Pediatrics 83:153-160
9. Silvasi DL, Rosen D, Rosen KR (1989) Absorption of midazolam by the scimed membrane oxygenator (abstract). Anesth Analg 68:S261

10. Rosen KR, Rosen DA, Silvasi DL (1989) Factors which affect midazolam uptake by the scimed membrane oxygenator (abstract). Anesth Analg 68:\$238

11. Tverskoy M, Fleyshman G, Ezry J, Bradley EL Jr, Kissin I (1989) Midazolam-morphine sedative interaction in patients. Anesth Analg 68:282-285

Dr. D. A. Rosen

Section of Pediatric Anesthesiology

C4139 Med Inn Bridge, Box 0800

University of Michigan Medical Center

1500 E. Medical Center Drive

Ann Arbor, MI 48109-0800

USA 